\title{
Consistency of students' conceptions of wave propagation: Findings from a conceptual survey in mechanical waves
}

\author{
Apisit Tongchai, ${ }^{1}$ Manjula Devi Sharma, ${ }^{2}$ Ian D. Johnston, ${ }^{2}$ Kwan Arayathanitkul, ${ }^{3}$ and Chernchok Soankwan ${ }^{3}$ \\ ${ }^{1}$ Institute for Innovative Learning, Mahidol University, Bangkok, Thailand \\ ${ }^{2}$ School of Physics, University of Sydney, Sydney, NSW, Australia \\ ${ }^{3}$ Department of Physics, Mahidol University, Bangkok, Thailand
}

(Received 24 November 2009; revised manuscript received 2 February 2011; published 22 July 2011)

We recently developed a multiple-choice conceptual survey in mechanical waves. The development, evaluation, and demonstration of the use of the survey were reported elsewhere [A. Tongchai et al., Developing, evaluating and demonstrating the use of a conceptual survey in mechanical waves, Int. J. Sci. Educ. 31, 2437 (2009)]. We administered the survey to 902 students from seven different groups ranging from high school to second year university. As an outcome of that analysis we were able to identify several conceptual models which the students seemed to be using when answering the questions in the survey. In this paper we attempt to investigate the strength with which the students were committed to these conceptual models, as evidenced by the consistency with which they answered the questions. For this purpose we focus on the patterns of student responses to questions in one particular subtopic, wave propagation. This study has three main purposes: (1) to investigate the consistency of student conceptions, (2) to explore the relative usefulness of different analysis techniques, and (3) to determine what extra information a study of consistency can give about student understanding of basic concepts. We used two techniques: first, categorizing and counting, which is widely used in the science education community, and second, model analysis, recently introduced into physics education research. The manner in which categorizing and counting is used is very diverse while model analysis has been employed only in prescriptive ways. Research studies have reported that students often use their conceptual models inconsistently when solving a series of questions that test the same idea. Our results support their conclusions. Moreover, our findings suggest that students who have had more experiences in physics learning seem to use the scientifically accepted models more consistently. Further, the two analysis techniques have different advantages and disadvantages. Our findings show that model analysis can be used in more diverse ways, provides flexibility in analyzing multiple-choice questions, and provides more information about consistency and inconsistency of student conceptions. An unexpected finding is that studying waves in other contexts (for example, quantum mechanics or electromagnetism) leads to more consistent answers about mechanical waves. The suggestion is that studying more abstract topics may solidify students' understanding of more concrete waves. While this might be considered to be intuitive, we have not actually found direct empirical studies supporting this conjecture.

DOI: 10.1103/PhysRevSTPER.7.020101

PACS numbers: 01.40.Fk

\section{INTRODUCTION}

An important observation that has been made by science educators for many years is that students, even those who can pass examinations easily, often have difficulty in understanding scientific concepts at a fundamental, conceptual level. This has led, over the past three decades, to much education research into the kind of mental constructions which students and subject experts use to organize their thinking about a scientific concept. It is found that the mental constructions employed by experts are much the same from one to another. So it is possible to construct a

Published by the American Physical Society under the terms of the Creative Commons Attribution 3.0 License. Further distribution of this work must maintain attribution to the author(s) and the published article's title, journal citation, and DOI. kind of average, which may be called the orthodox conception. On the other hand, students sometimes hold persistent conceptions which are internally self-consistent, but quite inconsistent with the orthodox understanding of the material; these are referred to as alternative conceptions [1-5]. Other terms can be found in the literature, such as conceptual models, mental models, and schemas. All these are sometimes given slightly different meanings. In this paper we will use the term "conceptual model" to stand for the more general mental constructions of which "alternative conception" is one example.

A popular response on the part of researchers has been to develop conceptual surveys aimed at uncovering specific alternative conceptions [6-9]. Many of these surveys take the form of multiple-choice tests, and that has inevitably led to attention being paid to the interpretation of answers to multiple-choice questions and how the information 
gained from them can be used in the classroom $[10,11]$. Many teachers would agree that a good way to test whether students "really understand" a concept is to ask the same question several times, in different contexts. The underlying belief is that, if a student answers those questions consistently correctly, he or she should be able to correctly answer all questions dealing with the same concept. What is being addressed here is the consistency of the student's conceptions. Below we give a more careful definition of what we mean by consistency.

There has been much physics education research effort devoted to this aspect of student understanding, and many studies have revealed that students solve problems across different contexts inconsistently [12-20]. The same is true of other discipline areas. Clough and Driver [12] interviewed students aged $12-16$ on problems concerning inheritance and natural selection and found that they used different conceptions in response to parallel questions, and that contextual features of the tasks seemed to be influencing their responses. More elaborate studies [3] applied the "substance schema" [2] to investigate student understanding of sound. They concluded that students' conceptions were consistent with a substance schema only in some respects, and that not all responses were accounted for by the schema. The understanding of density and pressure by students aged 12-15 was investigated using written questionnaires on different tasks with results indicating that students changed their reasoning across different questions, influenced by phenomenological features of the tasks [20].

In all these diverse projects, there seem to be several slightly different interpretations of what exactly the word "consistency" means, and how it should be measured. In our current project, being done within the subject area of physics, we identify (at least) two different interpretations of the kind of consistency we are interested in. The first interpretation simply concentrates on how systematically a student uses their conceptual understanding in answering questions that target the same concept, but in different contexts. The second interpretation concerns itself with the responses of a whole class of students and asks, on average, how well-definedly the class can be divided into the holders of various alternative conceptions. In this paper we address both of these interpretations.

As one example, based on responses in the area of force and motion (the most common topics taught in physics), the consistency of students' understanding was investigated using a series of open-ended questions. The responses were then categorized and counted using a computer program to uncover any predominant patterns in the answers [13]. A similar technique has also been used with multiple-choice questions [14] and with multiplechoice questions on both force and electricity [21]. We consider this kind of approach to be more or less equivalent to taking the first interpretation above. The categorizing and counting technique has also been extended to investigate how university students' understandings shift from one context to another [19]. On the other hand, Bao and Redish [22] have developed a technique called model analysis to investigate students' conceptual models. To date, it has been used only in the physics education community $[22,23]$. We consider this approach to line up better with the second interpretation.

Lastly, although there have been many research studies on student alternative conceptions across different discipline areas in physics, mechanical waves have been given relatively little attention. Yet studies have revealed that students also encounter a range of difficulties with conceptual understanding in that topic [24-33]. To complement this work, we developed and implemented a specially designed conceptual survey called the Mechanical Waves Conceptual Survey. In the first paper [34] describing this work we identified alternative conceptions held by students, and the way that they changed over time. In this paper we explore the consistency of those responses in the two interpretations we have described, using the two different analysis methods mentioned above. A brief description of each analysis method and data used to illustrate advantages and issues related to each method will be provided in later sections.

\section{PURPOSE OF THE STUDY}

The aims of the study were (1) to investigate the consistency of students' responses to sets of multiple-choice questions about the concepts of wave propagation, (2) to explore the relative usefulness of two different analysis techniques for analyzing these responses, categorizing and counting and model analysis, and (3) to determine what the consistency of students' responses can tell us about their understanding of the fundamental physics concepts involved.

\section{RESEARCH METHODOLOGY}

\section{A. Instrument}

The Mechanical Waves Conceptual Survey was developed iteratively involving trials with a total of 902 students from senior high school to second year university students [34]. The complete survey may be found in [35]. It covers four main subtopics: propagation, superposition, reflection, and standing waves. In this paper we focus only on the

TABLE I. Concepts covered in the survey on the subtopic of propagation.

\begin{tabular}{ll}
\hline \hline Question & \multicolumn{1}{c}{ Concept } \\
\hline 2,3 & Speed of sound waves \\
4,5 & Speed of waves on strings \\
$6,7,8$ & Displacement of medium \\
\hline \hline
\end{tabular}


TABLE II. Summary of participants.

\begin{tabular}{llrl}
\hline \hline Rank & Groups & $n$ & Descriptions \\
\hline 1 & 1stFund & 123 & First year fundamental physics students at a university in Australia. \\
2 & SydHigh & 54 & Australian senior high school students in Sydney. \\
3 & ThaiHigh & 270 & Thai senior high school students in Bangkok. \\
4 & 1 stReg & 287 & First year regular physics students at a university in Australia. \\
5 & 2ndReg & 48 & Second year regular physics students at a university in Australia. \\
6 & 1 1stAdv & 69 & First year advanced physics students at a university in Australia. \\
7 & 2ndAdv & 51 & Second year advanced physics students at a university in Australia. \\
\hline \hline
\end{tabular}

responses to the seven questions concerned with wave propagation. Table I shows the concepts covered. The full text of these seven questions is reproduced in the appendix.

\section{B. Participants}

The participants were seven different groups of students. They ranged from high schools both in Australia and Thailand, through three levels of first year university, fundamental, regular, and advanced, to second year university [34]. A brief overview is provided in Table II.

The groups of students were ranked not only according to the number of years of formal studies they had done, but also taking account of other kinds of informal instruction in physics they might have experienced (like PhysicsOlympiad involvement, special projects, and so on). We chose to use the phrase "previous engagement with physics learning" to refer to this mix of quantity and quality of education. From this perspective we ranked the groups of students as in Table II. This ranking was justified a posteriori by the results in our earlier study [34].

\section{Data analysis}

In this section, we provide brief descriptions of the two analysis techniques: categorizing and counting and model analysis. Both techniques were applied to the same pairs or groups of multiple-choice questions on the same test.

\section{Categorizing and counting}

This method of analysis is widely used. For example, Palmer [14] generated eight multiple-choice questions testing the same concept, but in different contexts. He categorized whether students were using the correct conception or the predominant alternative conception "motionimplies-force." He then counted the number of students who used the correct conception on all eight questions, or seven questions, and so on, while the number of students who invoked "motion-implies-force" were also counted on all eight questions, or seven questions, and so on. The resulting table was then able to assign a numerical value to the consistency with which students used either the correct conception or the predominant alternative conception. A similar technique was used in two topic areas, force and electricity, allowing for several alternative conceptions depending on the contexts of the questions [21]. Generalizing this scheme, the technique effectively makes the idealization that each student either (1) understands the concept completely (i.e., holds the orthodox conception), (2) holds some other alternative conception, or (3) holds an alternative conception other than those listed above, and/or seems to be guessing the answers.

Each of these is characterized by a specific pattern of responses to the survey questions. If any one student can be unambiguously put into one of these three classifications, on the basis of their selection of multiple-choice options, one might then be considered to be using their understanding completely consistently.

The categorizing and counting method of analysis is flexible and readily adaptable to suit different study designs with a firm grounding in alternative conceptions. The focus in such studies is on trends and not on statistical significance testing. We have adapted these analysis techniques to reveal consistency of students' responses to two questions testing the same concept. Students' responses were categorized and counted, and the results are presented in a later section.

\section{Model analysis}

This approach is based on the idea that when a student answers one of the questions on the test, they draw upon an underlying knowledge structure or mental model. In many cases it is possible to identify which particular models students are using when they answer individual questions, and there can be several different models which can be used by students to answer the questions. This technique effectively considers a class of students to be made up of (1) those employing the orthodox mental models, (2) those employing the common alternative mental model, (3) those employing no structured model, or just guessing the answers.

The model analysis technique does not concentrate on whether a student answer is correct or not; it is only interested in which model they are using. We give a brief description of model analysis here.

Consider a set of multiple-choice questions (total number $m$ ), where each choice of each question can be assigned 
to one or the other of these three "models." The response of any one student to the set of questions can be specified by a set of three numbers, which can be written as

$$
n_{1}^{k}, n_{2}^{k}, n_{3}^{k}
$$

where $k$ is an index denoting which student it is (out of a total number $N$ ). Clearly, assuming that the student answered all questions in the set,

$$
n_{1}^{k}+n_{2}^{k}+n_{3}^{k}=m .
$$

By way of interpretation, if $n_{1}^{k}=m$ and $n_{2}^{k}=n_{3}^{k}=0$, the $k$ th student has answered all questions using the orthodox mental model (model 1). Likewise, if $n_{2}^{k}=m$, that student has used model 2 (the most commonly held alternative mental model). And if $n_{3}^{k}=m$, the student (ideally) seems not to have used any structured mental model at all (model 3). But at least, in each of these examples, the student has answered consistently. On the other hand, if any two of these numbers are nonzero, the student has not been consistent. For instance, if $n_{1}^{k} \times n_{2}^{k} \neq 0$, the student is demonstrating confusion between models 1 and 2, sometimes using one and sometimes the other.

A difficulty faced by workers in this field is to find a representation to display these data in such a way that the interpretations described above are easy to see. The representation proposed by Bao [36] borrows from the formalism of quantum statistical mechanics. Using the three numbers, $n_{1}^{k}, n_{2}^{k}, n_{3}^{k}$, it constructs a $(3 \times 3)$ matrix, defined by

$$
D_{k}=\frac{1}{m}\left(\begin{array}{ccc}
n_{1}^{k} & \sqrt{n_{1}^{k} n_{2}^{k}} & \sqrt{n_{1}^{k} n_{3}^{k}} \\
\sqrt{n_{2}^{k} n_{1}^{k}} & n_{2}^{k} & \sqrt{n_{2}^{k} n_{3}^{k}} \\
\sqrt{n_{3}^{k} n_{1}^{k}} & \sqrt{n_{3}^{k} n_{2}^{k}} & n_{3}^{k}
\end{array}\right)
$$

This matrix represents a single student's model state, i.e., how the pattern of the student's responses to all questions spreads out over the three models. It is known as a single student model density matrix. It must be stressed that this definition contains no more information than is contained in the original three numbers themselves, which can be seen to lie along the main diagonal of the matrix. What it does do, however, is to make explicit any confusion between models, which is shown by the presence of offdiagonal elements.

The power of this representation is seen when we attempt to determine an average response to the set of questions for all students. We simply sum over all single student responses (and normalize) to obtain the model density matrix of the class:

$$
D=\frac{1}{N} \sum_{k=1}^{N} D_{k}=\frac{1}{N \cdot m} \sum_{k=1}^{N}\left(\begin{array}{ccc}
n_{1}^{k} & \sqrt{n_{1}^{k} n_{2}^{k}} & \sqrt{n_{1}^{k} n_{3}^{k}} \\
\sqrt{n_{2}^{k} n_{1}^{k}} & n_{2}^{k} & \sqrt{n_{2}^{k} n_{3}^{k}} \\
\sqrt{n_{3}^{k} n_{1}^{k}} & \sqrt{n_{3}^{k} n_{2}^{k}} & n_{3}^{k}
\end{array}\right)
$$

Each element of the class model density matrix represents different aspects of the students' understanding. The diagonal elements (which range from 0 to 1 ) represent the average number of responses which drew upon each of the three models. The off-diagonal elements (ranging from 0 to 0.5 ) tell us whether or not the students drew upon those models consistently (on average) or whether they jumped around between models.

As a brief guide to interpretation, consider the following hypothetical class model density matrices:

$$
\begin{aligned}
D_{\mathrm{I}} & =\left(\begin{array}{lll}
1 & 0 & 0 \\
0 & 0 & 0 \\
0 & 0 & 0
\end{array}\right), \\
D_{\mathrm{II}} & =\left(\begin{array}{ccc}
0.5 & 0 & 0 \\
0 & 0.3 & 0 \\
0 & 0 & 0.2
\end{array}\right), \\
D_{\mathrm{III}} & =\left(\begin{array}{ccc}
0.5 & 0.2 & 0.1 \\
0.2 & 0.3 & 0.1 \\
0.1 & 0.1 & 0.2
\end{array}\right) .
\end{aligned}
$$

$D_{\text {I }}$ shows that all students in the class answered all questions using model $1 . D_{\mathrm{II}}$ shows that $50 \%$ of the class used model 1 consistently, $30 \%$ used model 2 consistently, and $20 \%$ used model 3 consistently. $D_{\text {III }}$ shows that students answered using all models inconsistently; i.e., each student sometimes used model 1 , sometimes model 2 , and sometimes model 3. A comparison of $D_{\mathrm{II}}$ and $D_{\mathrm{III}}$ shows the key feature of the representation. In both cases, the average numbers for each model are the same (the diagonal elements), but the responses were consistent in $D_{\text {II }}$ and inconsistent in $D_{\mathrm{III}}$.

In our study, we applied this analysis technique to illustrate the consistency of students' responses to two sets of questions. It should be noted that model analysis is capable of more than this. By calculating the eigenvectors and eigenvalues of the class model density matrix, Bao [36] was able to assign a numerical value to the degree of inconsistency. We will not follow down that path since we are not interested in comparing pre-test and post-test scores. We simply want to make visual where the inconsistencies occur. The results are presented in Sec. IV B. 


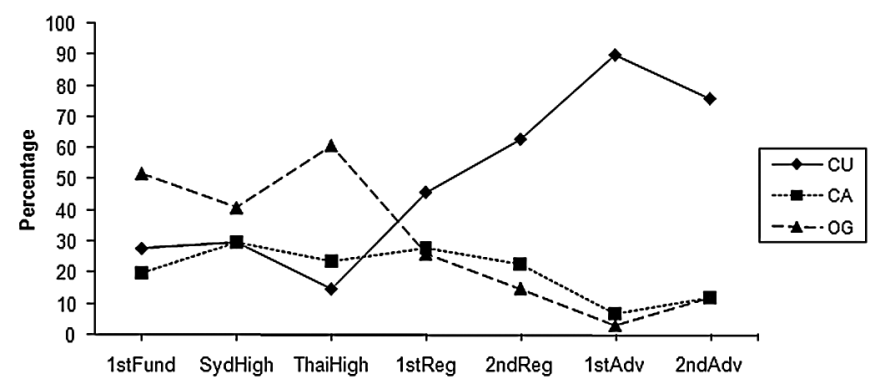

FIG. 1. The percentages of students' responses obtained using the categorizing and counting technique are plotted against previous engagement with physics learning (ranked as in Table II) for questions 2 and 3 on the speed of sound.

\section{RESULTS}

\section{A. Categorizing and counting}

1. Speed of sound waves in air--Students' responses to questions 2 and 3 on the speed of sound waves were placed into the three categories described below.

Complete understanding $(C U)$. - The answers to both questions demonstrate that the speed of sound waves depends on the properties of the air, a consistent and correct response.

Common alternative conception (CA).-The answers to both questions demonstrate that the speed of sound waves depends on their frequency, a consistent but incorrect response. This is the predominant common alternative conception $[28,31]$.

Other ideas or guessing $(O G)$.- The answers are not from the patterns above. Students' responses reflect a diverse range of alternative conceptions and there is no consistency between the answers to the two questions.

The percentages of students' responses in each category were counted and plotted against previous engagement with physics learning (ranked as in Table II) as shown in Fig. 1. As students' previous engagement increases, we note that the percentage of students in the category complete understanding, which represents consistently correct responses, increases to about $80 \%-90 \%$. (There are some fluctuations about the general trend, but we do not consider these to be significant.) Remember that the later year students would not have explicitly studied this material for several semesters; therefore, there seems to be a suggestion that deeper understanding of mechanical waves occurs as students cover a range of related subjects like quantum mechanics and electromagnetism. On the other hand, the common alternative conception persists at about $25 \%$ and then reduces quite sharply. The main switch going from novices to more experienced groups appears to be from other ideas $(\mathrm{OG})$ to complete understanding $(\mathrm{CU})$.

2. Concept of the speed of waves on strings.-Questions 4 and 5 are about the speed of wave pulses traveling along strings. The scientifically accepted concept is that the speed of waves depends on the properties of the string

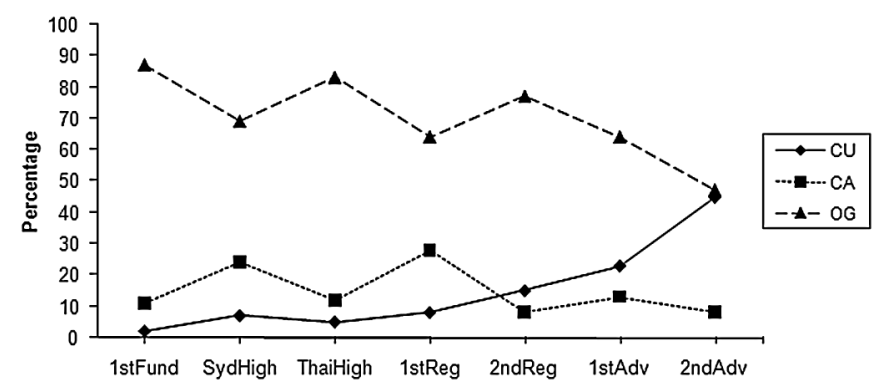

FIG. 2. The percentages of students' responses obtained using the categorizing and counting technique are plotted against previous engagement with physics learning (ranked as in Table II) for questions 4 and 5 on the speed of waves on strings.

only. The common alternative conception was that the speed of waves on strings depends on frequency. Results using the same set of categories (CU, CA, and OG) are shown in Fig. 2.

This pair of questions seems to be more difficult than the previous pair, resulting in a very different looking graph. The complete understanding group is very small at the start. It rises steadily as previous engagement with physics increases, but it is still only 50\% at second year university level. This is consistent with the fact that the scientifically accepted conception involved is actually counterintuitive. Most naive observations of waves on strings seem to support the idea that the wave speed does not depend only on the properties of the medium, but also on how the wave is generated.

The common alternative conception, that wave speed depends on frequency, does not attract many adherents at any level. On the other hand, the other ideas or guessing group is quite large at the start, about $80 \%$. This falls steadily as previous engagement with physics learning increases, but it is still 50\% at second year university level. This behavior is not characteristic of the usual mixture of ill-understood ideas or guesses. Instead, it almost looks like a third, consistent but incorrect, conception. It seems to warrant more research being done to find out exactly what the conception is, and special care being taken to change it.

\section{B. Model analysis}

We applied the technique model analysis to two sets of questions, each based on one particular concept. The first set consists of all the questions concerned with the speed of waves: questions $2,3,4$, and 5. The second set contains questions 6,7 , and 8 , concerned with two different aspects of the displacement of the medium. Results and explanations of each set of questions are shown below. We note that model analysis is being used with alternative conceptions rather than the more cognitive-type mental models they have been previously applied to.

1. Speed of waves.-Students' responses to questions 2, 3,4 , and 5 can be categorized into three models. 
TABLE III. Models and multiple-choice options for questions about wave speed.

\begin{tabular}{lccc}
\hline \hline Question & Model S1 & Model S2 & Model S3 \\
\hline 2 & $\mathrm{a}$ & $\mathrm{b}$ & $\mathrm{c}, \mathrm{d}$ \\
3 & $\mathrm{c}$ & $\mathrm{b}$ & $\mathrm{a}, \mathrm{d}$ \\
4 & $\mathrm{f}$ & $\mathrm{b}$ & $\mathrm{a}, \mathrm{c}, \mathrm{d}, \mathrm{e}$ \\
5 & $\mathrm{a}, \mathrm{b}$ & $\mathrm{d}$ & $\mathrm{c}$ \\
\hline \hline
\end{tabular}

Model S1: The answers demonstrate the understanding that the speed of waves depends on medium properties (the scientifically accepted conception).

Model S2: The answers are built on the notion that the speed of waves depends directly on frequency (common alternative conception).

Model S3: Other ideas or guessing.

Note, however, that there is not necessarily any one-toone mapping between models and answers. It is perfectly possible for two or more multiple-choice options to "belong" to one model. For example, question 5 option a (use a lighter string, under the same tension, because the velocity increases as the density decreases) and option b (use a heavier string, under the same tension, because the velocity increases as the density increases) are both in accordance with model S1. The associations of multiple-choice options with each model for all questions are shown in Table III.

Table IV shows the class model density matrices of all groups of students. Remember that the diagonal elements of each matrix represent the proportion of students' responses which are based on the three models. Overall, it is clear that as the previous engagement with physics learning increases, the trend of the consistent use of conceptual models (diagonal elements) shifts from other ideas (model S3) and common alternative conception (S2) to the scientifically accepted model (S1).

Further, students with low previous engagement with physics learning seem to use all models equally. Indeed, they use all models inconsistently, as shown by the high values of the off-diagonal elements. At the other end of the table, the most advanced students tend to use only the scientifically accepted model, and do so consistently.

We also plotted the diagonal elements of all density matrices against the ranked previous physics learning, generating Fig. 3. This shows that a lot more students seem to use the scientifically accepted model after they have more experience in physics learning. At a superficial level, Figs. 1 and 3 look similar. However, students who

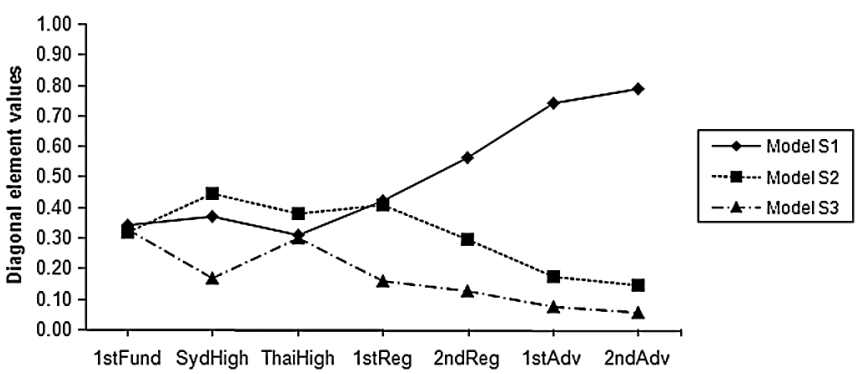

FIG. 3. Trend of the diagonal elements of all class model density matrices plotted against previous engagement with physics learning (ranked as in Table II) for questions $2-5$ on wave speed.

showed "complete understanding" in Figs. 1 and 2 (solid line) can only fall into the model S1 group in Fig. 3. Likewise, those who chose the "common alternative" (dotted line) fall into model S2. But the "other ideas or guessing" (dashed line) can fall into any of the three models. Therefore it is not easy to see the relationship between these graphs, particularly Figs. 2 and 3, even though they are constructed from some of the same data. To our way of thinking, the matrices in Table IV are the easiest to interpret.

2. Displacement of the medium.-Questions 6, 7, and 8 are concerned with displacement of the medium. Using this set of questions we will demonstrate that model analysis can be applied to analyzing the same set of questions more than once depending on which particular fundamental concept we are interested in. For this set of questions we are interested in two main concepts: (i) that sound waves are longitudinal, rather than transverse waves (models L), and (ii) that the particles of the medium oscillate about a central position, rather than keep moving in the same direction (models $\mathrm{O}$ ). The analysis method and the results of the two sets of questions are shown below.

(i) Sound waves as longitudinal waves.-Students' responses to this set of questions can be categorized into three models.

TABLE V. Models and multiple-choice options for questions about longitudinal waves.

\begin{tabular}{lccc}
\hline \hline Question & Model L1 & Model L2 & Model L3 \\
\hline 6 & b, d & c & a, e \\
7 & b, c, f, g & d, e & a, h \\
8 & b, c, f, g & d, e & a, h \\
\hline \hline
\end{tabular}

TABLE IV. Class model density matrices for questions 2-5 on wave speed.

\begin{tabular}{|c|c|c|c|c|c|c|c|c|c|c|c|c|c|c|c|c|c|c|c|c|}
\hline \multicolumn{3}{|c|}{ 1stFund $(n=123)$} & \multicolumn{3}{|c|}{ SydHigh $(n=54)$} & \multicolumn{3}{|c|}{ ThaiHigh $(n=270)$} & \multicolumn{3}{|c|}{1 stReg $(n=287)$} & \multicolumn{3}{|c|}{2 ndReg $(n=48)$} & \multicolumn{3}{|c|}{ 1stAdv $(n=69)$} & \multicolumn{3}{|c|}{$2 \operatorname{ndAdv}(n=51)$} \\
\hline 0.34 & 0.17 & 0.19 & 0.38 & 0.22 & 0.15 & 0.31 & 0.22 & 0.19 & 0.43 & 0.20 & 0.14 & 0.57 & 0.19 & 0.13 & 0.75 & 0.15 & 0.12 & 0.79 & 0.12 & 0.09 \\
\hline 0.17 & 0.32 & 0.17 & 0.22 & 0.45 & 0.14 & 0.22 & 0.3 & 0.20 & 0.20 & 0.41 & 0.11 & 0.19 & 0.30 & 0.07 & 0.15 & 0.17 & 0.03 & 0.12 & 0.15 & 0.01 \\
\hline 0.19 & 0.17 & 0.33 & 0.15 & 0.14 & 0.17 & 0.19 & 0.20 & 0.30 & 0.14 & 0.11 & 0.16 & 0.13 & 0.07 & 0.13 & 0.12 & 0.03 & 0.08 & 0.09 & 0.01 & 0.06 \\
\hline
\end{tabular}


TABLE VI. Average class density matrices for questions 6-8 on longitudinal waves.

\begin{tabular}{|c|c|c|c|c|c|c|c|c|c|c|c|c|c|c|c|c|c|c|c|c|}
\hline \multicolumn{3}{|c|}{ 1stFund $(n=123)$} & \multicolumn{3}{|c|}{ SydHigh $(n=54)$} & \multicolumn{3}{|c|}{ ThaiHigh $(n=270)$} & \multicolumn{3}{|c|}{1 stReg $(n=287)$} & \multicolumn{3}{|c|}{ 2ndReg $(n=48)$} & \multicolumn{3}{|c|}{ 1stAdv $(n=69)$} & \multicolumn{3}{|c|}{2 ndAdv $(n=51)$} \\
\hline 0.56 & 0.14 & 0.13 & 0.72 & 0.05 & 0.07 & 0.50 & 0.11 & 0.17 & 0.71 & 0.06 & 0.06 & 0.81 & 0.03 & 0.06 & 0.88 & 0.05 & 0.02 & 0.86 & 0.04 & 0.03 \\
\hline 0.14 & 0.26 & 0.09 & 0.05 & 0.19 & 0.01 & 0.11 & 0.26 & 0.07 & 0.06 & 0.20 & 0.04 & 0.03 & 0.12 & 0.02 & 0.05 & 0.09 & 0.00 & 0.04 & 0.10 & 0.00 \\
\hline 0.13 & 0.09 & 0.18 & 0.07 & 0.01 & 0.09 & 0.17 & 0.07 & 0.24 & 0.06 & 0.04 & 0.10 & 0.06 & 0.02 & 0.08 & 0.02 & 0.00 & 0.03 & 0.03 & 0.00 & 0.05 \\
\hline
\end{tabular}

TABLE VII. Models and the multiple-choice options for questions about oscillations.

\begin{tabular}{lccc}
\hline \hline Question & Model O1 & Model O2 & Model O3 \\
\hline 6 & b, c & d & a, e \\
7 & b, c, d, e & f, g & a, h \\
8 & b, c, d, e & f, g & a, h \\
\hline \hline
\end{tabular}

Model L1: The answers demonstrate that a sound wave is a longitudinal wave; i.e., the motion of the dust particle is forwards or backwards along a line (the scientifically accepted conception).

Model L2: The answers are built on the notion that a sound wave is a transverse wave; i.e., the dust particle moves up and down (common alternative conception).

Model L3: Other ideas or guessing.

The associations of multiple-choice options in each model for all questions are generated as shown in Table V.

Table VI shows the class model density matrices for models L1, L2, and L3 of all groups of students. Consider first the diagonal elements. As the previous engagement with physics learning increases, students' responses tend to shift from models L2 and L3 to the scientifically accepted model (model L1). We note that the scientifically accepted model is highly used (over $50 \%$ ) by all groups, suggesting that students do indeed know that sound waves are longitudinal waves. Secondly, the off-diagonal element values seem to decrease as the previous engagement with physics learning increases, suggesting that students progressively use their conceptions more consistently as they have studied more physics.

(ii) Patterns of dust particle movement.-For the next fundamental concept, we are interested in students' conceptions of whether the dust particle oscillates or moves in a fixed direction. Again, students' responses can be categorized into three models.

Model O1: The answers demonstrate that the dust particle oscillates about a central position (the scientifically accepted conception).
Model O2: The answers are built on the notion that the dust particle does not oscillate, but moves along a line (common alternative conception).

Model O3: Other ideas or guessing.

The associations of multiple-choice options in each model for all questions are generated as shown in Table VII, and the class model density matrices are shown in Table VIII.

Similar patterns emerge in both diagonal and offdiagonal elements as seen in Table VIII. We can now compare across Tables VII and VIII. This comparison reflects different ways of interpreting the same set of responses. One is about the longitudinal waves, and the other is about oscillation. Regarding this particular fundamental physics, students seem to start with no clear idea at all about how particles move, and by the time they get to second year physics, $86 \%-95 \%$ of responses have a conceptual model including both aspects of the motion that they can use consistently.

\section{DISCUSSION AND CONCLUSION}

This project dealt with the development and implementation of the specially designed survey, the Mechanical Waves Conceptual Survey. The results presented in the previous paper [34] allowed us to identify several student alternative conceptions, and we were able to describe how these alternative conceptions changed over time. But there are always questions of consistency with a survey like this. How can we be sure that students' responses genuinely reflect what they are thinking?

This paper used the same data, this time focusing on the consistency of the responses. In this process we came up against issues in three main areas. Firstly, there was the issue of flexibility of the different analysis techniqueswhat can they be used to analyze and what type of useful interpretations will result from their use? Secondly, there were technical issues regarding what sort of information the different analysis techniques could give. Thirdly, there was an examination of what the consistency of students' responses told us about their understanding of the fundamental physics concepts involved.

TABLE VIII. Average class density matrices for questions 6-8 on oscillations.

\begin{tabular}{|c|c|c|c|c|c|c|c|c|c|c|c|c|c|c|c|c|c|c|c|c|}
\hline \multicolumn{3}{|c|}{ 1stFund $(n=123)$} & \multicolumn{3}{|c|}{ SydHigh $(n=54)$} & \multicolumn{3}{|c|}{ ThaiHigh $(n=270)$} & \multicolumn{3}{|c|}{1 stReg $(n=287)$} & \multicolumn{3}{|c|}{2 ndReg $(n=48)$} & \multicolumn{3}{|c|}{ 1stAdv $(n=69)$} & \multicolumn{3}{|c|}{$2 \operatorname{ndAdv}(n=51)$} \\
\hline 0.39 & 0.12 & 0.11 & 0.65 & 0.02 & 0.06 & 0.50 & 0.12 & 0.15 & 0.65 & 0.05 & 0.06 & 0.71 & 0.08 & 0.04 & 0.83 & 0.01 & 0.02 & 0.95 & 0.01 & 0.03 \\
\hline 0.12 & 0.43 & 0.10 & 0.02 & 0.26 & 0.03 & 0.12 & 0.27 & 0.09 & 0.05 & 0.25 & 0.04 & 0.08 & 0.22 & 0.04 & 0.01 & 0.14 & 0.00 & 0.01 & 0.01 & 0.00 \\
\hline 0.11 & 0.10 & 0.18 & 0.06 & 0.03 & 0.09 & 0.15 & 0.09 & 0.24 & 0.06 & 0.04 & 0.10 & 0.04 & 0.04 & 0.08 & 0.02 & 0.00 & 0.03 & 0.03 & 0.00 & 0.05 \\
\hline
\end{tabular}




\section{A. Investigating consistency}

We find that, in the area of mechanical waves, as in other areas, students respond inconsistently. Students start using some ideas more consistently earlier in their studies than others. For example, whether sound waves are transverse or longitudinal seems to be understood earlier than oscillations. If a survey can be used to diagnose such features, then teachers can respond in real time.

We found that, in general, student conceptual understanding increased with increasing previous engagement with physics. If we remember that the survey addressed some basic concepts not explicitly covered in higher years but definitely used, the indication is that as students continue studying and using these concepts in other topics, they improve their understanding of these ideas. For example, studying quantum mechanics and electromagnetism seems to deepen their understanding of basic mechanical waves. On the other hand, a better understanding of basic waves can scaffold a sound understanding of quantum mechanics and electromagnetism. Most teachers would agree. There is no doubt that more research needs to be carried out in such areas, and the study of consistency in students' answers is a gateway for such studies.

\section{B. Analysis techniques}

In the first instance, when looking at the different analysis techniques, we found that each had different strengths and weaknesses. The categorizing and counting technique is a flexible, simple, and convenient analysis tool for evaluating students' responses and is easy to use in a classroom. It is used to investigate trends with no reliance on further statistical analysis. Furthermore, it is also relatively easy to interpret its results. On a very basic level, this technique reflects the assumption that "you only understand completely if you can answer the same question in the same way in different contexts."

There is one assumption which is implicit in these techniques: if students have the correct conceptual model, they will answer the question correctly. But we know that students are not necessarily consistent. They frequently seem to hold several different conceptual models in their heads, which they switch between when answering survey questions. The model analysis technique confronts this problem head-on. In this study model analysis has been used with alternative conceptions rather than mental models for which they were specifically designed. Our results demonstrate that model analysis is more robust than anticipated.

We used this technique to analyze four questions relating to the speed of waves and three questions on displacement of the medium. The technique produced meaningful information which agreed with similar data given by the other technique. However, it also gave us further information about how consistently students used their conceptions, which we can see from the off-diagonal elements of the matrices. Moreover, the technique was applied to analyzing different aspects of the same questions specified by which student conceptions we are interested in. Despite the fact that model analysis is mathematically more complicated to use, it proved to be flexible in analyzing welldeveloped multiple-choice conceptual surveys.

\section{Consistency and conceptual understanding}

The results from the two different techniques agreed in suggesting that students do indeed use their conceptions inconsistently across different contexts. For instance, in questions 2 and 3, students seemed to switch easily between the scientifically accepted conception and the common alternative. These findings support studies found in the literature $[12,14,21]$.

Considering Figs. 1-3, they all agree on one thing, namely, that the scientifically accepted responses increased with increasing previous engagement with physics. Similarly, the figures show that the alternative conceptions and other ideas all decreased. However, the data illustrated in Fig. 2 require some comment. In this figure a large number of responses fall into the "other ideas or guessing" category. Furthermore, they are persistent, suggesting that this category is a complete conceptual model in its own right. At the lowest end of the previous physics experience spectrum, some $90 \%$ of students hold this "model," and at the high end it is still held by $50 \%$. One suggestion is that the students did not recognize that the questions were related to one another. Seemingly, they were independent, meaning that students did not draw on the same knowledge base in order to answer them. So it is understandable that there will be a large degree of inconsistency.

In our earlier paper, question 4 was investigated independently and in great detail. The most common alternative conception (that wave speed depends on frequency) was extremely obvious (see Fig. 7 in [34]). In this present paper, we looked specifically at how consistently students applied their conceptual models, using the categorizing and counting technique. They only scored (CU) if they got both questions 4 and 5 correct. This scoring eroded considerably the number who seemed to be using the alternative conception consistently. These findings suggest that, in order to identify student conceptual models, it is not enough simply to consider how they answer individual questions. It is also necessary to determine how consistently they answer sets of questions.

Lastly, we have analyzed the same three questions twice. First, using conceptual models based on longitudinal waves (Table V) and second based on particle oscillations (Table VII). We find that the naïve students have a slightly better understanding of longitudinal waves than oscillations. However, the most experienced students seem to demonstrate the reverse, better understanding of oscillations and poorer understanding of longitudinal waves. We speculate this is because these students have studied 
general wave motion in their later years, but not sound waves explicitly. How formal instruction influences this is interesting and needs to be further explored.

\section{IMPLICATIONS}

Both techniques have their usefulness and were able to show that the student conceptual understanding increases as they studied more physics from high school to second year university level. The second technique was particularly useful in highlighting inconsistencies in the way students answered the questions. In terms of classroom practices, it is important to be careful when interpreting students' responses to this kind of survey. They may apparently get the right answers, but still be confused about the basic concepts. The more sophisticated model analysis technique gives a readily visual summary of where the students are mixing conceptual models.

\section{ACKNOWLEDGMENTS}

The authors wish to thank students and staff who participated in the study. The authors would also like to thank Sydney University Physics Education Research group, the Physics Education Network of Thailand, the Institute for Innovative Learning, Mahidol University, and the Institute for Promotion of Teaching Science and Technology, Thailand. We would like to thank the reviewers for their insightful comments that have clarified our thoughts and the paper.

\section{APPENDIX: QUESTIONS FROM THE MECHANICAL WAVES CONCEPTUAL SURVEY WHICH ARE USED IN THIS STUDY}

Consider the following description and answer questions 2-3.

Students $X$ and $Y$ are standing $50 \mathrm{~m}$ apart and yell "Yo!" at each other at exactly the same time.

(2) They yell at each other with the same loudness, but $Y$ yells with a higher pitch than $X$ does. Who will hear the other's sound first?

a. They will hear each other at exactly the same time because the speed of sound waves depends on the properties of the air.

b. $X$ will hear the sound first because the speed of sound waves depends on frequency according to $v=f \lambda$.

c. They will hear each other at exactly the same time because the speed of sound waves depends on amplitude.

d. $X$ will hear the sound first because sound with a higher frequency is more penetrating.

(3) Student $Y$ yells louder than $X$, but they yell at each other with the same pitch. Who will hear the other's sound first? a. $X$ will hear the sound first because the speed of the waves depends on the amplitude of the sound.

b. They will hear each other at exactly the same time because the speed of the waves depends on the frequency according to $v=f \lambda$.

c. They will hear each other at exactly the same time because the speed of the waves depends on the properties of the air.

d. $X$ will hear the sound first because the wave with the larger amplitude travels further.

Consider the following description and answer questions 4-5.

One end of a long taut string is tied to a distant pole while the other end of the string is held by a girl (see figure below). This girl quickly flicks her hand up and down to create a pulse moving towards the pole.

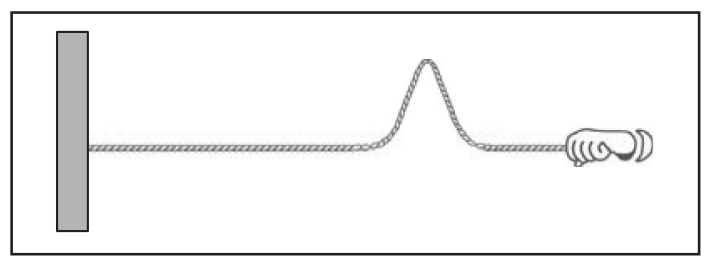

(4) She now wants to produce a pulse that takes a shorter time to reach the pole. How can she do this?

a. Flick the string harder to push more force into the pulse.

b. Flick the string faster to create a pulse with higher frequency.

c. Flick the string further up and down to create a pulse with larger amplitude.

d. Flick the string a shorter distant up and down to create a pulse with smaller amplitude.

e. Wait until the first pulse is reflected back then flick again to add the pulses together.

f. None of the above would produce a pulse that takes a shorter time to reach the pole.

(5) She still wants the pulse to reach the pole in a shorter time by changing the properties of the string. How can she do this?

a. She should use a lighter string, under the same tension, because the velocity increases as the density decreases.

b. She should use a heavier string, under the same tension, because the velocity increases as the density increases.

c. She should decrease the tension in the string because the velocity increases as the tension decreases.

d. None of the above would produce a pulse that takes a shorter time because the speed is determined by frequency and wavelength according to $v=f \lambda$. 
Consider the following description and answer questions 6-8.

A dust particle hovers in front of a silent loudspeaker (see figure below). The loudspeaker is turned on and plays a loud tone at a constant pitch.

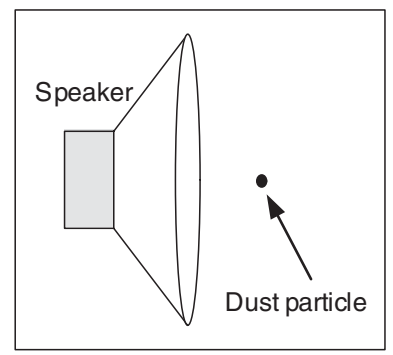

(6) How will the dust particle move?

a. It will stay in the same position.

b. It will move back and forth about the same position.

c. It will move up and down about the same position. d. It will move away from the speaker.

e. It will move away as a sine curve.

For questions 7 and 8, choose the description from "a" through " $h$ " which best answers each question.

a. It will stay at the same position.

b. It will move back and forth further.

c. It will move back and forth faster.

d. It will move up and down further.

e. It will move up and down faster.

f. It will move away further.

g. It will move away faster.

h. It will move away faster as a sine curve.

(7) The pitch of the sound is increased but the volume stays the same. What happens to the motion of the dust particle?

(8) The volume of the sound is increased but the pitch stays the same. What happens to the motion of the dust particle?
[1] J.S. Lee, Exploring students' understanding concerning batteries-Theories and practices, Int. J. Sci. Educ. 29, 497 (2007).

[2] M. Reiner, J. D. Slotta, M. T. H. Chi, and L.B. Resnick, Naïve physics reasoning: A commitment to substancebased conceptions, Cogn. Instr. 18, 1 (2000).

[3] H. Eshach and J. L. Schwartz, Sound stuff? Naïve materialism in middle-school students' conceptions of sound, Int. J. Sci. Educ. 28, 733 (2006).

[4] A. G. Harrison, D. J. Grayson, and D.F. Treagust, Investigating a grade 11 student's evolving conceptions of heat and temperature, J. Res. Sci. Teach. 36, 55 (1999).

[5] I. D. Johnston, K. Crawford, and P. R. Fletcher, Students' difficulties in learning quantum mechanics, Int. J. Sci. Educ. 20, 427 (1998).

[6] D. F. Treagust, Evaluating students' misconceptions by means of diagnostic multiple choice items, Res. Sci. Educ. 16, 199 (1986).

[7] R. K. Thornton and D.R. Sokoloff, Assessing student learning of Newton's laws: The Force and Motion Conceptual Evaluation and the Evaluation of Active Learning Laboratory and Lecture Curricula, Am. J. Phys. 66, 338 (1998).

[8] D. P. Maloney, T. L. O'Kuma, C. J. Hieggelke, and A. Van Heuvelen, Surveying students' conceptual knowledge of electricity and magnetism, Am. J. Phys. 69, S12 (2001).

[9] S. Wuttiprom, M. D. Sharma, I. D. Johnston, R. Chitaree, and C. Soankwan, Development and use of a conceptual survey in introductory quantum physics, Int. J. Sci. Educ. 31, 631 (2009).

[10] T. I. Smith and M.C. Wittmann, Applying a resources framework to analysis of the Force and Motion
Conceptual Evaluation, Phys. Rev. ST Phys. Educ. Res. 4, 020101 (2008).

[11] B. W. Frank, S.E. Kanim, and L.S. Gomez, Accounting for variability in student responses to motion questions, Phys. Rev. ST Phys. Educ. Res. 4, 020102 (2008).

[12] E. E. Clough and R. Driver, A study of consistency in the use of students' conceptual frameworks across different task contexts, Sci. Educ. 70, 473 (1986).

[13] M. Finegold and P. Gorsky, Students' concepts of force as applied to related physical systems: A search for consistency, Int. J. Sci. Educ. 13, 97 (1991).

[14] D. Palmer, How consistently do students use their alternative conceptions?, Res. Sci. Educ. 23, 228 (1993).

[15] D. Palmer, Students' application of a biological concept: Factors affecting consistency, Res. Sci. Educ. 26, 409 (1996).

[16] R. Tytler, Consistency of children's use of science conceptions: Problems with the notion of "conceptual change," Res. Sci. Educ. 24, 338 (1994).

[17] J. R. Watson, T. Prieto, and J.S. Dillon, Consistency of students' explanations about combustion, Sci. Educ. 81, 425 (1997).

[18] L. Webb and I. Morrison, The consistency of primary children's conceptions about the Earth and its gravity, Sch. Sci. Rev. 81, 99 (2000).

[19] R. Warnakulasooriya and L. Bao, in Proceeding of the Physics Education Research Conference, Madison, WI, 2003, edited by J. Marx, S. Franklin, and K. Cummings (AIP, New York, 2004), p. 109.

[20] G. Fassoulopoulos, P. Kariotoglou, and P. Koumaras, Consistent and inconsistent pupils' reasoning about intensive quantities: The case of density and pressure, Res. Sci. Educ. 33, 71 (2003). 
[21] P. Licht and G. D. Thijs, Method to trace coherence and persistence of preconceptions, Int. J. Sci. Educ. 12, 403 (1990).

[22] L. Bao and E. F. Redish, Model analysis: Representing and assessing the dynamics of student learning, Phys. Rev. ST Phys. Educ. Res. 2, 010103 (2006).

[23] L. Bao, K. Hogg, and D. Zollman, Model analysis of fine structures of student models: An example with Newton's third law, Am. J. Phys. 70, 766 (2002).

[24] Z. Hrepic, Identifying students' mental models of sound propagation, Master's thesis, Kansas State University, 2002.

[25] L. C. Linder, University physics students' conceptualizations of factors affecting the speed of sound propagation, Int. J. Sci. Educ. 15, 655 (1993).

[26] L. Maurines, Spontaneous reasoning on the propagation of visible mechanical, Int. J. Sci. Educ. 14, 279 (1992).

[27] T. R. Rhoads and R. J. Roedel, in Proceeding of the 28th Annual Frontiers in Education Conference, Tempe, Arizona, 1999, http://www.foundationcoalition.org/ publications/journalpapers/fie1999/1245.pdf.

[28] C.M. Wittmann, R.N. Steinberg, and E.F. Redish, Making sense of how students make sense of mechanical waves, Phys. Teach. 37, 15 (1999).
[29] C. M. Wittmann, The object coordination class applied to wave pulses: Analysing student reasoning in wave physics, Int. J. Sci. Educ. 24, 97 (2002).

[30] C. M. Wittmann, Understanding and affecting student reasoning about sound waves, Int. J. Sci. Educ. 25, 991 (2003).

[31] A. Tongchai, K. Arayathanitkul, and C. Soankwan, in Proceedings of the 2007 National UniServe Conference, Sydney, 2000, http://sydney.edu.au/science/uniserve_ science/pubs/procs/2007/37.pdf.

[32] S. K. Sengoren, R. Tanel, and N. Kavcar, Drawings and ideas of physics teacher candidates relating to the superposition principle on a continuous rope, Phys. Educ. 41, 453 (2006).

[33] M.E. Houle and G. M. Barnett, Students' conceptions of sound waves resulting from the enactment of a new technology-enhanced inquiry-based curriculum on urban bird communication, J. Sci. Educ. Tech. 17, 242 (2008).

[34] A. Tongchai, M.D. Sharma, I. D. Johnston, K. Arayathanitkul, and C. Soankwan, Developing, evaluating and demonstrating the use of a conceptual survey in mechanical waves, Int. J. Sci. Educ. 31, 2437 (2009).

[35] http://www.physics.usyd.edu.au/super/mwcs/mwcs.pdf.

[36] L. Bao, Dynamics of student modeling: A theory, algorithms, and application to quantum mechanics, Ph.D. thesis, University of Maryland, 1999. 